Gastroenterology, Bradford Teaching Hospital, Bradford, UK; ${ }^{6}$ Department of Gastroenterology, Central Manchester University Hospital NHS foundation Trust, Manchester, UK; ' Department of Surgery, Royal Infirmary, Glasgow, Glasgow, UK; ${ }^{8}$ Department of Gastroenterology, Adednbrooke's Hospital, Cambridge, Cambridge, UK; ${ }^{9}$ Department of Gastroenterology, Royal Infirmary, Edinburgh, UK; ${ }^{10}$ Department of Histopathology, University College Hospital, London, UK

Introduction Squamous Cell Cancer (SCC) of the oesophagus has a poor prognosis with 5 -year survival at $10 \%$. Squamous high grade dysplasia (HGD) is the precursor lesion to SCC. Risk of progression to SCC with Squamous HGD can be $65 \%$ at 5 years. Radiofrequency ablation (RFA) is a minimally invasive ablation technique with proven efficacy for early neoplasia in Barrett's Oesophagus.

Methods Prospective multicenter registry of patients undergoing RFA for Squamous HGD and early carcinoma in situ (CIS) from eight UK centers. Nodular lesions were removed by endoscopic mucosal resection before RFA. Treatment consisted of a single ablation at $12 \mathrm{~J} / \mathrm{cm}^{2}$. Patients were followed-up 3 monthly and repeat biopsies taken. Those with residual dysplasia underwent RFA 3 months later until 12 months where they were assessed for treatment success or failure.

Results 25 patients have undergone ablation for squamous HGD/ CIS. We report on 17 patients to have completed protocol. Mean length of dysplastic epithelium ablated was $5 \mathrm{~cm}(1-14)$. Mean time to protocol completion was 8.7 months. CR-HGD was seen in $59 \%$ of patients and CR-D in 47\% at end of protocol biopsy with mean of 1.4 RFA treatments (1-3). All those with successful outcomes remain free of dysplasia at most recent biopsy, median follow-up 10.6 months (2-36) from first treatment. At protocol completion, six of 17 patients (35\%) had progressed to invasive cancer and referred for surgery or chemo-radiotherapy. Three patients $(18 \%)$ required dilatations for oesophageal structuring after first treatment. Two of these patients have required serial dilatations thereafter with an average of four dilatations per patient.

Conclusion Squamous HGD and CIS are very aggressive pathologies as evidenced by the fact a third of patients progressed to invasive disease despite RFA. The role of RFA in these patients remains unclear. In our series $47 \%$ of patients responded to RFA \& have reassuringly remained free of dysplasia at last follow-up. These figures are lower than limited published data to date but in our series an emphasis was placed on restaging carefully after each treatment to assess for progression. As our experience grows with confidence in identifying these lesions more accurately and increasing the frequency and number of ablations administered over the protocol period, dysplasia reversal rates will be expected to increase.

Competing interests None declared.

\section{PWE-028 HALO RADIOFREQUENCY ABLATION FOR HIGH GRADE DYSPLASIA AND EARLY MUCOSAL NEOPLASIA ARISING IN BARRETT'S OESOPHAGUS: INTERIM RESULTS FORM THE UK HALO RADIOFREQUENCY ABLATION REGISTRY}

doi:10.1136/gutjnl-2012-302514d.28

${ }^{1} \mathrm{R}$ J Haidry, ${ }^{*}{ }^{1} \mathrm{~J}$ Dunn, ${ }^{2} \mathrm{M}$ Banks, ${ }^{1} \mathrm{~A}$ Gupta, ${ }^{1} \mathrm{M}$ A Butt, ${ }^{3} \mathrm{H}$ Smart, ${ }^{4} \mathrm{P}$ Bhandari, ${ }^{5} \mathrm{~L}-\mathrm{A}$ Smith, ${ }^{6} \mathrm{R}$ Willert, ${ }^{7} \mathrm{G}$ Fullarton, ${ }^{8} \mathrm{M}$ Di Pietro, ${ }^{9} \mathrm{I}$ Penman, ${ }^{10} \mathrm{H}$ Barr, ${ }^{11} \mathrm{C}$ Gordon, ${ }^{12} \mathrm{P}$ Patel, ${ }^{12} \mathrm{P}$ Boger, ${ }^{13} \mathrm{~N}$ Kappor, ${ }^{14} \mathrm{~B}$ Mahon, ${ }^{15} \mathrm{M}$ Burnell, ${ }^{16} \mathrm{M}$ Novelli, ${ }^{12} \mathrm{~L}$ B Lovat. ${ }^{1}$ Department of Surgery, National Medical Laser Centre, London, UK; ${ }^{2}$ Department of Gastroenterology, University College Hospital, London, UK; ${ }^{3}$ Department of Gastroenterology, Royal Liverpool University Hospital, Liverpool, UK; ${ }^{4}$ Department of Gastroenterology, Princess Alexandra Hospital, Portsmouth, UK; ${ }^{5}$ Department of Gastroenterology, Bradford Teaching Hospital, Bradford, UK; ${ }^{6}$ Department of Gastroenterology, Central Manchester University Hospital NHS foundation Trust, Manchester, UK; ${ }^{7}$ Department of Surgery, Royal Infirmary, Glasgow, Glasgow, UK; ${ }^{8}$ Department of Gastroenterology, Adednbrooke's Hospital, Cambridge, Cambridge, UK; ${ }^{9}$ Department of Gastroenterology, Royal Infirmary, Edinburgh, UK; ${ }^{10}$ Department of Surgery, Gloucestershire Hospitals NHS Trust, Gloucester, UK; ${ }^{11}$ Department of Gastroenterology, Royal Bournemouth Hospital, Bournemouth, UK; ${ }^{12}$ Department of Gastroenterology,
Southampton University Hospital, Southampton, UK; ${ }^{13}$ Department of Gastroenterology, Aintree University Hospital, Liverpool, UK; ${ }^{14}$ Department of Gastroenterology, Queen Elizabeth hospital, Birmingham, UK; ${ }^{15}$ Department of Biostatistics, University College London, London, UK; ${ }^{16}$ Department of Histopathology, University College Hospital, London, UK

Introduction Barrett's oesophagus (BE) is the pre-cursor to oesophageal adenocarcinmoa (OAC). High grade dysplasia (HGD) and early mucosal neoplasia in BE has historically been treated with surgery. Recently there is a shift towards minimally invasive endotherapy with endoscopic mucosal resection (EMR) and Radiofrequency ablation (RFA).

Methods Prospective registry from 14 UK centers to audit RFA outcomes in patients with HGD and early neoplasia in BE. Prior to RFA, any visible lesions were first removed by EMR. Patients then underwent RFA 3 monthly until all visible BE was ablated or cancer developed. Biopsies were taken at the end of this protocol.

Results 216 patients have completed protocol, mean age 68.6 years (40-90), 81\% male. Mean time to protocol end 11.3 months (IOR 8-14.3), median 2 ablations and mean of $2.4(2-6)$ during protocol with mean 1.4 circumferential ablations and 1.2 focal ablations performed during protocol. Mean length BE segment ablated is $5.8 \mathrm{~cm}$ (1-20). CR-HGD was achieved in $83 \%$ patients at protocol end biopsy. CR-D was $76 \%$ and CR-BE $50 \%$ at this point. CR-D was more likely in short segment BE $(<5 \mathrm{~cm})$ at protocol end ( $82 \%$ vs $54 \%$, $\mathrm{p}<0.0001$, Fisher's exact test). Patients who required EMR during RFA protocol were less likely to achieve CR-D than those who had RFA alone ( $52 \%$ vs $79 \%, p=0.002$, fishers exact test). $3.7 \%$ patients progressed to invasive cancer at protocol end. Complications include one perforation and $1 \%$ incidence of superficial tears. 37 patients have at least 12 months or more follow-up after successful completion of protocol (range 12-42), median 16.2 months. Durability in these is excellent with $95 \%$ dysplasia free at most recent biopsy.

Conclusion This is the largest series to date of patients undergoing RFA from 14 UK centers. End of protocol CR-D is satisfactory at $76 \%$ and successful eradication appears to be durable. Patients with short segment BE are likely to respond better. Our data represent real life outcomes of integrating minimally invasive endotherapy into demanding endoscopy service commitments.

Competing interests None declared.

\section{PWE-029 OBJECTIVE ASSESSMENT OF PHYSICAL ACTIVITY AS A MEASURE OF FUNCTIONAL RECOVERY AND QUALITY OF LIFE FOLLOWING OESOPHAGO-GASTRIC CANCER RESECTION}

doi:10.1136/gutjnl-2012-302514d.29

R J E Skipworth, ${ }^{*}$ P Hendry, S Paterson-Brown, K C Fearon. University of Edinburgh, Royal Infirmary of Edinburgh, Edinburgh, UK

Introduction Functional recovery following surgery is determined by the interaction between pre-operative performance, post-operative catabolism, nutritional status, and mood. Physical activity (PA) is an important domain of health-related quality-of-life (HROL), and may be a useful objective index of recovery. We aimed to use an accelerometer-based activity metre (ActivPAL) to monitor post-operative $\mathrm{PA}$ in oesophago-gastric (OG) cancer patients undergoing surgery with curative intent.

Methods PA measures, including step count, time spent in various body positions, and energy expenditure of activity, were assessed over 7-day periods in patients undergoing oesophagectomy or gastrectomy $(n=16)$. Nutritional status, HRQL (FAACT, FACIT-F and EORTC-OLQC30 questionnaires), and mood (HADS questionnaire) were also assessed. Time-points were pre-operatively and 1-2 weeks, 5-6 weeks, 3 months and 6 months post-operatively.

Results Compared with pre-operative results, PA measures were decreased by $23-89 \%(p<0.05) 1-2$ weeks post-operatively, and were still decreased by $15-57 \% \quad(p<0.05) \quad 5-6$ weeks 
post-operatively. At 3 months, all PA measures except time spent upright $(p=0.009)$ and time spent standing $(p=0.013)$ had recovered. Measures of PA correlated positively with physical and functional domains of HROL, including EORTC-OLO30 Global Health Status, FAACT Trial Outcome Index (TOI) and FACIT-TOI $(p<0.001)$, and inversely with HADS-Depression $(p<0.001)$.

Conclusion There is marked impairment of $\mathrm{PA}$ at the time of hospital discharge and a gradual recovery over 3-6 months. This carries significant implications in a disease where surgical patients may survive $<2$ years. PA measures are suitable outcomes for evaluating the impact of enhanced recovery programmes on functional recovery and HROL.

Competing interests None declared.

\section{PWE-030 IMMUNOCYTOCHEMICAL ASSESSMENT OF INTRA-TUMOUR MICROVESSEL DENSITY IN OESOPHAGOGASTRIC CANCER DOES NOT HAVE PROGNOSTIC SIGNIFICANCE}

doi:10.1136/gutjnl-2012-302514d.30

${ }^{1} \mathrm{R}$ T Gray, ${ }^{*}{ }^{1} \mathrm{M}$ E O'Donnell, ${ }^{2} \mathrm{~J}$ A McGuigan, ${ }^{1} \mathrm{G}$ M Spence. ${ }^{1}$ Upper Gastrointestinal Surgery, The Ulster Hospital, Belfast, UK; ${ }^{2}$ Thoracic Surgery, Royal Victoria Hospital, Belfast, UK

Introduction Intra-tumour microvessel density (IMD), a marker of tumour angiogenesis, correlates with metastasis and poor prognosis in many cancers. In oesophagogastric cancer however, the prognostic significance of IMD assessment remains incompletely investigated. Methods Patients undergoing surgery with curative intent, without pre-operative chemotherapy, were prospectively recruited between February 1999 and August 2000. Immunocytochemical staining of tumour microvessels was undertaken using anti-CD34 (OBEND 10 clone) antibodies. IMD (microvessels per $\mathrm{mm}^{2}$ ) was assessed using a validated "hot-spot" technique. Patients were followed-up over a 10year period using the Northern Ireland Cancer Registry. The relationship between IMD and standard clinicopathological variables was assessed using the Mann-Whitney $U$ test. Univariate survival analysis was calculated using a Cox's proportional hazard model while survival analysis was calculated using Kaplan-Meier estimation and log rank. Results 61 patients were recruited (male $=45$ ) with a median age of 66.0 years (range 39-83). The overall 10-year survival rate was $19.7 \%(n=12)$. IMD was significantly higher in males compared to females (332.93 vs 252.44, $\mathrm{p}=0.04$ ) and adenocarcinomas compared to squamous cell carcinomas (356.10 vs 203.66, $\mathrm{p}<0.001)$. On univariate survival analysis only lymphovascular invasion predicted poor prognosis (HR 2.26, 95\% CI 1.01 to 5.07, $p=0.05$ ). Kaplan-Meier survival analysis demonstrated no difference in longterm survival for patients with IMD levels greater or less than the median value (738 days vs 882 days, $p=0.67$ ).

Conclusion Immunocytochemical analysis of IMD does not have a prognostic benefit in determining long-term survival in patients with oesophagogastric cancer.

Competing interests None declared.

\section{PWE-031 THE POTENTIAL OF HAEM TRANSPORT PROTEINS AS THERAPEUTIC TARGETS IN THE TREATMENT OF OESOPHAGEAL ADENOCARCINOMA}

doi:10.1136/gutjnl-2012-302514d.31

${ }^{1} \mathrm{~S}$ J Ford, ${ }^{* 1} \mathrm{M}$ Bedford, ${ }^{2} \mathrm{O}$ Tucker, ${ }^{3} \mathrm{D}$ Alderson, ${ }^{1} \mathrm{~T}$ H Iqbal, ${ }^{1} \mathrm{C}$ Tselepis. ${ }^{1}$ Cancer Studies, University of Birmingham, Birmingham, UK; ${ }^{2}$ Upper GI Surgery, Birmingham, UK; ${ }^{3}$ Cancer Studies, University Hospital Birmingham, Birmingham, UK

Introduction Epidemiological evidence supports the potential role of dietary haem iron in gastrointestinal carcinogenesis. ${ }^{12}$ Oesophageal cancer cells acquire inorganic and organic iron by progressive upregulation of iron and haem transport proteins. ${ }^{3}$ These alterations result in increased cellular iron loading which is likely to drive cellular proliferation. ${ }^{3}$ The effect of perturbing haem import proteins, hemopexin receptor (LRP1) and haem carrier protein (HCP1), on cancer cell iron transport, phenotype and tumour burden is unknown. The aim was to determine the in vitro and in vivo effects of depriving oesophageal cancer cells of haem iron.

Methods Stable knock-downs of LRP1 and HCP1 were created by infection with specific shRNA lenti-viral vectors. Successful knockdown was confirmed by Western blotting. The effect on cellular iron transport and cell phenotype was assessed by qRT-PCR and phenotypic experiments for viability, proliferation, migration and anchorage independent growth. Stable knock-downs of LRP1 and HCP1 were then xenografted into NOD-SCID mice as an in vivo model of oesophageal adenocarcinoma.

Results Perturbation of LRP1 and HCP1 caused a compensatory upregulation of inorganic iron import proteins and a decrease in iron storage capacity. Neoplastic activity was significantly impaired compared to control (proliferation, viability, colony forming and migration - all $p=<0.01$ ). Loss of active haem iron import significantly reduced xenograft tumour burden in murine models with a $70 \%(p=0.014)$ and $58 \%(p=0.05)$ reduction in average xenograft weight compared to control.

Conclusion Haem import significantly contributes to iron loading in oesophageal cancer cells and creates a more aggressive phenotype. Functional inhibition of haem importing proteins LRP1 and HCP1 curbs neoplastic activity and significantly reduces in vivo tumour burden in murine models. Haem iron transport proteins are potential therapeutic targets in the treatment of oesophageal adenocarcinoma.

Competing interests None declared.

\section{REFERENCES}

1. Cross AJ, Freedman ND, Ren J, et al. Meat consumption and risk of esophageal and gastric cancer in a large prospective study. Am J Gastroenterol 2011:106:432-42.

2. Cross AJ, Leitzmann MF, Gail MH, et al. A prospective study of red and processed meat intake in relation to cancer risk. PLoS Med 2007;4:e325.

3. Boult J, Roberts K, Brookes MJ, et al. Overexpression of cellular iron import proteins is associated with malignant progression of esophageal adenocarcinoma. Clin Cancer Res 2008;14:379-87.

\section{PWE-032 ENDOSCOPIC MUCOSAL RESECTION (EMR) FOLLOWED BY ADJUVANT RADIOFREQUENCY ABLATION (RFA) CAN RESULT IN BETTER OUTCOMES COMPARED TO EMR ALONE IN PATIENTS WITH BARRETT'S EARLY NEOPLASIA (EN). A COMPARATIVE STUDY FROM A TERTIARY CENTRE IN THE UK}

doi:10.1136/gutjnl-2012-302514d.32

${ }^{1} \mathrm{~S}$ Sami, ${ }^{1} \mathrm{E}$ Telakis, ${ }^{1} \mathrm{~J}$ Mannath, ${ }^{2} \mathrm{P}$ Kaye, ${ }^{1} \mathrm{~K}$ Ragunath. ${ }^{1}$ Department of Gastroenterology, Nottingham Digestive Diseases Centre and NIHR Biomedical Research Unit, Nottingham University Hospitals NHS Trust, Nottingham, UK; ${ }^{2}$ Department of Histopathology, Nottingham University Hospitals NHS Trust, Nottingham, UK

Introduction RFA has shown efficacy in eradicating Barrett's EN (high grade dysplasia (HGD) or intra-mucosal cancer (IMC)). To our knowledge, there are no studies directly comparing outcomes in patients with EN who undergo EMR alone vs EMR followed by RFA. The aim of this study was to assess the efficacy, safety and long term outcomes of adjuvant RFA in this setting.

Methods We searched our prospective Barrett's Oesophagus EMR database for patients who had EMR of lesions harbouring EN followed by RFA for eradication of residual Barrett's mucosa between 2007 and 2008 as part of a multi-centre trial (intervention group). The control group included patients with similar lesions 